\title{
LA OBLIGACIÓN DE INFORMAR Y EL CONSENTIMIENTO INFORMADO EN ODONTOLOGÍA: UNA MIRADA CRÍTICA MÁS ALLÁ DE LA REGULACIÓN NORMATIVA ${ }^{1}$
}

\author{
Estefanía Esparza-Reyes ${ }^{2}$, Víctor Beltrán Varas ${ }^{3}$, Pamela Beltrán Varas ${ }^{4}$
}

Resumen: El presente trabajo tiene por finalidad evidenciar que la regulación normativa chilena de la obligación de informar y el consentimiento informado en Odontología no resulta suficiente para un apropiado ejercicio de tales derechos. Para ello, describe en qué consisten tales obligaciones, considerando aspectos históricos y doctrinarios, y analiza el derecho a ser informado y el consentimiento informado, haciendo énfasis en su finalidad y utilidad. Con posterioridad detalla, de manera crítica y pormenorizada, la actual regulación chilena sobre la materia y aspectos de la práctica odontológica en materia de consentimiento informado. En último término propone soluciones para superar los inconvenientes encontrados.

Palabras clave: obligación de informar, consentimiento informado, derechos del paciente, odontología, regulación legal

Right to be informed and informed consent in Dentistry: A critical look beyond law regulation

Abstract: This article aims to make clear that the Chilean regulation of the right to be informed and informed consent in Dentistry is not sufficient for the proper exercise of such rights. To do this, it begins by describing this obligations, considering historical and doctrinal aspects. It continues to analyze the right to be informed and informed consent, emphasizing its purpose and usefulness. Subsequently, the current Chilean regulation on the matter is critically detailed, to finish exposing aspects of dental practice in the field of informed consent. Lastly, some solution proposals for overcoming the problems found are indicated.

Key words: right to be informed, informed consent, patient's rights, dentistry, law regulation

A obrigaçáo de informar e o consentimento informado em Odontologia: uma visáo crítica para além da regulaçáo normativa

Resumo: O presente trabalho tem por finalidade mostrar que a regulação normativa chilena da obrigação de informar e do consentimento informado em Odontologia não é suficiente para um exercício apropriado de tais direitos. Para isto, descreve no que consistem tais obrigaçóes, considerando aspectos históricos e doutrinários, e analisa o direito a ser informado e o consentimento informado dando ênfase na sua finalidade e utilidade. Além disto, detalha de maneira crítica e pormenorizada a atual regulação chilena sobre o tema e aspectos da prática odontológica em termos de consentimento informado. Por fim, propóe soluçốes para superar os inconvenientes encontrados.

Palavras chave: obrigação de informar, consentimento informado, direitos dos pacientes, odontologia, regulação legal

\footnotetext{
${ }^{1}$ Trabajo financiado parcialmente por la Universidad de La Frontera y parte del proyecto DIUFRO DI20-57.

${ }^{2}$ Comité Ético Científico de la Universidad de La Frontera. Departamento de Ciencias Jurídicas, Universidad de La Frontera, Temuco, Chile. ORCID: https://orcid.org/0000-0003-3374-2660

Correspondencia: estefania.esparza@ufrontera.cl

${ }^{3}$ Comité Ético Científico Universidad de La Frontera. Departamento de Odontología Integral Adultos, Universidad de La Frontera, Temuco, Chile. ORCID: https://orcid.org/0000-0001-6046-821X

Correspondencia: victor.beltran@ufrontera.cl

${ }_{4}^{4}$ Programa de Magíster en Derecho Administrativo, Facultad de Derecho, Universidad de Los Andes, Chile. ORCID: https://orcid. org/0000-0001-5786-1141

Correspondencia: pame.beltran@gmail.com.
} 


\section{Introducción}

Existe claridad de la existencia de la obligación de informar y del consentimiento informado por parte de los profesionales odontólogos. La normativa chilena aplicable establece los contenidos que deben incorporarse en dichos procesos; las consideraciones que debe tener el odontólogo, basado en las características de los pacientes, así como el proceso de reclamo en caso de no efectuarse de la manera indicada. Sin embargo, resulta interesante analizar varios tópicos de esta regulación, tales como si resulta apropiada, en la práctica, para la finalidad que se propone y si es concordante con los nuevos modelos de relación entre odontólogopaciente. De esta forma es posible cuestionarse sobre la temática y, eventualmente, proponer algunas vías de solución en caso de existir falta de concordancia. A ello se abocarán las siguientes líneas.

\section{Aspectos generales}

Para estudiar el origen de la obligación de informar y del consentimiento informado es posible remontarse al principio hipocrático de primum non nocere, vigente durante 25 siglos en Europa y que comenzó a tener aplicaciones específicas en el ámbito de la investigación en salud solo a partir del siglo XIX(1).

Con posterioridad a la Segunda Guerra Mundial, y particularmente influenciado por los experimentos científicos en campos de concentración, comenzaron a adoptarse disposiciones jurídicas generales, entre las que destaca el Código de $\mathrm{Nu}$ remberg, de 1947, que recoge principios básicos de la experimentación médica en seres humanos. Varios años más tarde se adoptaron nuevas disposiciones normativas, tales como la Declaración de Helsinki, de 1964, y el informe Belmont, de 1979, las cuales, recogiendo el mismo espíritu del Código de Nuremberg, plasmaron los principios de la bioética: no maleficencia (beneficencia), autonomía y justicia.

Ahora bien, pese a que existen registros anteriores a la Segunda Guerra Mundial, particularmente en Alemania y Estados Unidos de Norteamérica, de que las malas prácticas médicas no se limitaban a los ensayos clínicos, sino también a tratamien- tos médicos, tales como esterilizaciones forzadas, eutanasia, entre otros(2), lo cierto es que la preocupación parece haberse centrado en las investigaciones científicas, particularmente médicas, y no con el mismo empeño en relaciones terapéuticas propiamente tales.

En un sentido similar, existen antecedentes del reconocimiento de esta autonomía y derecho a ser informado, específicamente sobre el consentimiento informado en la relación médico-paciente. Esta vez no se trataba de disposiciones jurídicas generales, sino de casos concretos que llegaron a tribunales de justicia, en los cuales se alegaba la necesidad de informar a los pacientes, de modo que participasen de las decisiones terapéuticas(3).

No obstante los antecedentes antes mencionados, el impulso definitivo de la utilización del consentimiento informado y de la obligación de información se produjo en Estados Unidos de Norteamérica en los años sesenta o setenta, periodo que coincide con un gran desarrollo de la ciencia y de la bioética como disciplina(4).

Recién en 1973 se adoptó la primera Carta de derechos del paciente por parte de la Asociación Americana de Hospitales. Este documento reconoció diferentes derechos fundamentales, entre los que destaca el derecho del paciente a "obtener de su médico toda la información disponible relacionada con su diagnóstico, tratamiento y pronóstico, en términos razonablemente comprensibles para él". De igual manera, estableció la relación entre derecho a la información y consentimiento informado en los siguientes términos: "El paciente tiene derecho a que su médico le comunique todo lo necesario para que pueda dar su consentimiento informado previamente a la aplicación de cualquier procedimiento o tratamiento".

Unos años más tarde, en 1976 y 1981 respectivamente, la Asamblea Parlamentaria del Consejo de Europa recomendó y el Council on Judicial and Ethical Affairs de la American Medical Association declaró el derecho de los pacientes a ser informados para tomar sus propias decisiones(5). 


\section{El consentimiento informado y el deber de in- formar}

Durante años, en la relación médico-paciente primó el modelo paternalista. En este modelo, el profesional adoptaba las decisiones y actuaba según su criterio, pues la opinión del paciente no tenía importancia(6). Este enfoque entendía que concurrían ciertas circunstancias, tales como asimetría de saberes y capacidades, subordinación del actuar del paciente al saber del profesional de la salud y concepción del paciente como un sujeto ignorante y ausente de la responsabilidad de su enfermedad(7).

En la actualidad, donde haya un enfoque bioético es posible encontrar distintos modelos de consentimiento informado, tales como el informativo, el interpretativo, el deliberativo e, inclusive, el modelo instrumental(8).

Todos los actuales modelos tienen en común que se basan en la autonomía del paciente para tomar sus propias decisiones y en el reconocimiento de la igual dignidad de los seres humanos. Este reconocimiento no puede sino generar que el paciente sea corresponsable de las decisiones médicas, para lo cual se requiere que sea debidamente informado.

Ante la ausencia de definiciones de derecho a la información y de consentimiento informado, las distintas legislaciones establecen la información que debe entregarse a los pacientes en caso de requerir algún tipo de intervención o tratamiento, por lo que debe cumplirse con lo señalado en dichas normas. Sin embargo, es necesario destacar que, entre los autores, no hay completo acuerdo sobre qué aspectos comprende este derecho a ser informado, de modo que algunos consideran que solo es necesario informar sobre el diagnóstico, tratamiento y pronóstico(6), mientras que, para otros, debe indicarse la naturaleza del procedimiento, las alternativas y los riesgos(9).

El consentimiento informado, por su parte, ha sido definido como un acuerdo de voluntades entre profesional sanitario y paciente, el cual debe producirse en términos de conocimiento, capacidad y autonomía para tomar una decisión sobre la base de ciertas alternativas propuestas(10). Sin embargo, las definiciones más extendidas entienden que el consentimiento informado es un proceso en el cual el paciente es informado de los aspectos más relevantes de una intervención y/o tratamiento, y que finaliza con una toma de decisión por parte del paciente(11).

La toma de decisión del paciente suele plasmarse por escrito, circunstancia que ha generado gran confusión entre tales conceptos(12); sin embargo, no deben confundirse, pues el documento no es sino una formalidad de que el proceso se llevó a cabo y sirve para conocer en qué términos se realizó.

Ahora bien, el consentimiento informado no posee una única dimensión o visión. En primer término, es posible distinguir una dimensión filosófica, en cuanto entraña el ejercicio de la libertad de conciencia y desde aquí que posea una dimensión ética. En segundo lugar, posee una dimensión jurídico-técnica(9). De esta se derivan ciertas funciones que cumple el consentimiento informado, principalmente para un ordenamiento jurídico determinado. Por una parte, legitima una interferencia en la corporalidad del paciente, pero también asigna el riesgo de una intervención correctamente ejecutada al paciente(13). En un sentido similar, es posible afirmar que el consentimiento informado actúa acotando los riesgos respecto de los cuales ambas partes acuerdan interactuar y definiendo el objeto del contrato(14), pero, todavía más, puede actuar como prueba(12) de que efectivamente se cumplió con el deber de informar, de sus términos y de que el paciente estuvo de acuerdo con ellos.

Entre los autores no existe completo acuerdo acerca de qué debe contener y qué requisitos debe cumplir un consentimiento informado para considerarse válido. En efecto, así ha sido señalado en múltiples ocasiones: "en él no hay extremo que no esté plagado de cuestiones y problemas: quién debe informar, a quién se debe dar la información y sobre el qué, el cuándo, el cuánto y el cómo de lo que se debe informar" (15).

La selección de contenidos suele venir dada por las disposiciones legales, que habitualmente enumeran los datos que debe contener, así como los requisitos que deben cumplirse para que dicho 
documento tenga valor jurídico 5 .

Con todo, los autores han señalado algunos requisitos con los que debe contar un consentimiento informado para ser válido. Así, se ha indicado que debe comprender toda la información necesaria para que el paciente tome una decisión; debe ser voluntario, es decir, sin presiones; quien lo da debe ser completamente capaz, por lo que en caso de ser menor de edad o sin capacidad para decidir debe prestarlo el representante legal; debe otorgarlo la persona sobre la cual se realizará el acto médico; el propósito y la causa del consentimiento debe ser la recuperación de la salud; debe tener una forma externa, verbal o escrita y, por último, debe ser previo a la realización del acto(6). En otras ocasiones se ha entendido que debe incluirse información sobre qué se hará; los riesgos previsibles; los riesgos de baja ocurrencia pero alta materialidad; otras alternativas de tratamiento; las ventajas y desventajas tanto de alternativas de tratamiento como de los distintos procedimientos diagnósticos, entre otros(16).

Es necesario señalar que un consentimiento informado no posee una fórmula de estilo o un formato obligatorio, pues no existen parámetros objetivos(16). Ni siquiera es posible establecer de manera completa las menciones que debe contener. Esto se debe a que cada caso es único y requerirá en una dedicación pormenorizada a los aspectos más relevantes. Pero, en igual sentido, comprendiendo que la finalidad es tomar una decisión (personal) informada, los términos en que esta información se entregue dependerá de factores subjetivos, tales como la edad, nivel de comprensión y de preparación del paciente, pero también, del estado emocional en que se encuentre(12). Pese a lo anterior, en el afán de colaborar con las tareas de sus asociados, destaca a estos efectos que distintas instituciones profesionales propongan a sus asociados modelos tipo de dichos instrumentos, como el que proporciona el Colegio de Cirujano Dentistas de Chile A.G a los odontólogos colegiados ${ }^{6,7}$.

\footnotetext{
${ }^{5}$ En el siguiente epígrafe se abordarán los requisitos específicos, establecidos para Chile en la ley 20.584.

${ }^{6}$ Documento se encuentra disponible en http://www.colegiodentistas.cl/inicio/wp-content/uploads/2017/01/Información-de-Consentimiento-Informado.pdf. Última revisión 29 de abril de 2020.

${ }^{7}$ Es interesante destacar que, en circunstancias especiales, como es la
}

\section{La regulación del consentimiento informado en Odontología}

Con anterioridad a la dictación de Ley $\mathrm{N}^{\circ} 20.584$, que regula los derechos y deberes de las personas en relación con acciones vinculadas a su atención de salud, de 14 de abril de 2012, no existía normativa nacional sobre consentimiento informado en materia de acciones de salud.

La regulación del consentimiento informado se realizó durante muchos años a partir de principios y derechos establecidos en tratados internacionales y/o recomendaciones.

Actualmente en Chile, tal como ocurre en muchos países, no existe regulación completa sobre el deber de informar, ni sobre consentimiento informado en materia odontológica de manera específica.

Resulta aplicable a estos efectos la Ley 20.584, cuya última modificación se produjo en diciembre de 2019 y que contempla un Reglamento sobre entrega de información y expresión de consentimiento informado en las atenciones de salud. Dicho instrumento se encuentra contenido en el Decreto 31 del Ministerio de Salud ${ }^{8}$. La aplicación de esta ley y de este reglamento a la Odontología, pese a no estar expresamente señalado, se debe a que dicha normativa es aplicable a todas las profesiones reguladas por el Título $\mathrm{V}$ del Código Sanitario, en el que se indican normas específicas

atención odontológica en fase IV covid-19, el Colegio de Dentistas a través de sus redes sociales, comunicó el 7 de mayo de 2020 que solicitó aclaraciones y orientaciones a la Subsecretaría de Salud Pública respecto de la obligatoriedad de una firma de consentimiento informado específico, debido al riesgo de contagio durante la atención de los pacientes que son sujetos de garantías del sistema GES en sus problemas de salud odontológica. Fuente: https://twitter.com/i/web/ status/1258505657235333121. Última revisión 8 de mayo de 2020.

${ }^{8}$ Existen otros dos Reglamentos de la misma cartera que hacen alusión al deber de informar. El primero está contenido en el Decreto 38, que aprueba Reglamento sobre derechos y deberes de las personas en relación con las actividades vinculadas con su atención de salud, de 2012, y el Decreto 114, que aprueba reglamento de la Ley 20.120 sobre la investigación científica en el ser humano, su genoma, y prohíbe la clonación humana, de 2010. Ninguna de dichas disposiciones ha sido incluida en este trabajo debido a distintas razones. En el primer caso, pues se cuenta con un reglamento específico sobre el derecho a ser informado, mientras que el reglamento mencionado solo aborda la temática desde una perspectiva general; en el segundo, debido a que las disposiciones hacen alusión al deber de informar y al consentimiento en investigaciones científicas, lo que excede el objetivo del presente artículo. 
para el ejercicio de la Odontología. Tal afirmación es también posible de colegir de la lectura de su artículo $1^{\circ}$ ?.

Los principios orientadores de este cuerpo normativo se cimentan en la dignidad de las personas y su autonomía en la atención de salud; los derechos de las personas a decidir informadamente; el respeto a los menores de edad; el respeto de las personas en situación de enfermedad terminal; el respeto de la autonomía frente a la investigación científica; el respeto por las personas con discapacidad psíquica e intelectual; la confidencialidad de la información de salud; el reconocimiento del derecho de la participación ciudadana en salud y el marco legal para la tutela ética en los servicios asistenciales.

La primera alusión de la ley al derecho a ser informado se encuentra en una disposición referida al trato digno y respetuoso que debe brindarse a los pacientes, así, el artículo 5 letra a) establece como un deber de los prestadores de salud "Velar porque se utilice un lenguaje adecuado e inteligible durante la atención; cuidar que las personas que adolezcan de alguna discapacidad, no tengan dominio del idioma castellano o sólo lo tengan en forma parcial, puedan recibir la información necesaria y comprensible, por intermedio de un funcionario del establecimiento, si existiere, o con apoyo de un tercero que sea designado por la persona atendida". Como puede notarse, se trata de una vinculación estrecha entre la dignidad y la manera en que debe entregarse la información.

En segundo término ${ }^{10}$, el artículo 9 establece la

\footnotetext{
9 "Artículo $1^{\circ}$.- Esta ley tiene por objeto regular los derechos y deberes que las personas tienen en relación con acciones vinculadas a su atención de salud.

Sus disposiciones se aplicarán a cualquier tipo de prestador de acciones de salud, sea público o privado. Asimismo, y en lo que corresponda, se aplicarán a los demás profesionales y trabajadores que, por cualquier causa, deban atender público o se vinculen con el otorgamiento de las atenciones de salud".

${ }^{10}$ En estricto rigor, existen otras alusiones que pueden considerarse parte de este derecho a ser informado. Se trata de las obligaciones de los prestadores institucionales contenidas en el artículo 8 de la Ley, las cuales se refieren principalmente a aspectos de funcionamiento interno, tales como atenciones o tipos de atenciones que el profesional ofrece y sus valores; condiciones previsionales, trámites y documentos; reglamento interno del centro asistencial y formas de reclamo, comentarios, agradecimientos y sugerencias. Como puede notarse, estos aspectos se relacionan más bien con normas de funcionamiento y de naturaleza económica, por lo cual exceden el propósito del presente trabajo.
}

obligación de los miembros del equipo de salud de portar identificaciones, mientras que el artículo 10 se refiere al derecho a ser informado de manera específica.

Por una parte, dicha disposición contempla la primera característica de la información, la cual debe ser oportuna (con anterioridad al inicio de una intervención o tratamiento) y comprensible, entendiendo que puede ser brindada por el médico u otro profesional tratante (odontólogo). Indica, además que la información debe brindarse de manera confidencial y en un lugar apropiado, acto del que hace responsable al prestador de salud, sin distinguir si es público o privado.

Indica, por otra parte, el contenido de dicha información, a saber: el estado de salud; el posible diagnóstico de la enfermedad; las alternativas de tratamiento disponibles; los riesgos que puedan ocasionar dichas alternativas; el pronóstico esperado; el proceso previsible del postoperatorio si procediere. Como puede notarse, solo de una interpretación muy amplia de este artículo podría considerarse que, dentro de la información que debe suministrar el odontólogo, se encuentran, por ejemplo los componentes de las prótesis o materiales que se utilizan, hecho que podría afectar a pacientes que tienen algún tipo de contraindicación, por ejemplo religiosa, respecto de algunos elementos en particular.

La misma disposición introduce el elemento subjetivo del proceso de información, pues entiende que la información mencionada debe realizarse de acuerdo con la edad y condición personal y emocional del paciente. Esto quiere decir que la manera de comunicar, mas no el contenido de la información, debe ser (es un imperativo) de acuerdo no solo a la edad del paciente, sino a circunstancias personales, tales como el nivel educacional, el nivel de afectación emocional que presente, entre otras circunstancias.

Establece también la norma que, en caso de que a juicio del médico tratante (debe entenderse comprendido el odontólogo) el paciente se encuentre en una condición que no le permita recibir o entender la información directamente, dicha información debe ser dada a su representante legal o a la persona bajo cuyo cuidado se encuentre. Este 
último caso se refiere principalmente a menores de edad. Una vez que el paciente haya recuperado la conciencia y la capacidad de comprender debe ser informado de los aspectos y en la forma indicada con anterioridad. Es decir, en este caso solo cambia a quien se entrega la información preliminar y el momento en que la misma se le entrega al paciente, más el contenido y las demás características. Se debe actuar de una manera similar en en caso de tratarse de una atención de emergencia o urgencia, en la que, además, la imposibilidad de entregar dicha información no podrá, en ningún caso, dilatar o posponer tal atención.

El párrafo 7 de la misma ley, "De la autonomía de las personas en su atención de salud”, en sus artículos 14 y 15 , regula el consentimiento informado. Tales disposiciones consideran que el consentimiento informado es un derecho que posee el paciente.

El artículo 14 señala que toda persona tiene derecho a otorgar o denegar su voluntad para someterse a cualquier procedimiento o tratamiento de salud. Establece asimismo que este derecho posee las limitaciones comprendidas en el art. 16 de la misma Ley.

El mismo artículo señala la forma en que debe ser ejercido este derecho, de forma libre (sin presiones), voluntaria (sin interferencias), expresa (con una manifestación unívoca, sin que quepan dudas de la aceptación) e informada.

El artículo 14 señala que el proceso de consentimiento informado se efectúa generalmente de manera verbal. Sin embargo, impone la necesidad de escriturar la información misma; el hecho de haberla entregado y la aceptación o rechazo del paciente, en caso de intervenciones quirúrgicas, procedimientos diagnósticos y terapéuticos invasivos, y, en general, para la aplicación de procedimientos que conlleven un riesgo relevante y conocido para la salud del afectado.

Agrega igualmente el artículo 14 que, en ningún caso, el rechazo a tratamientos podrá tener como objetivo la aceleración artificial de la muerte, la realización de prácticas eutanásicas o el auxilio al suicidio.
El artículo 15, por su parte, indica situaciones excepcionales en las cuales no se requiere de manifestación de voluntad del paciente, aunque exige igualmente que se adopten las medidas apropiadas en orden a garantizar la protección de la vida.

Se trata de casos en los que la falta de aplicación de procedimientos, tratamientos o intervenciones suponen un riesgo para la salud pública, en los que debe dejarse constancia en la ficha clínica; casos en los que la condición de salud o cuadro clínico del paciente implique riesgo vital o secuela funcional grave de no mediar atención médica inmediata e impostergable, sin que el paciente pueda manifestar voluntad u obtener consentimiento de su representante legal, apoderado o persona a su cargo; y, por último, cuando la persona se encuentra en incapacidad de manifestar su voluntad y no es posible obtenerla de su representante legal, por no existir o por no ser habido.

La misma Ley, en el título cuarto sobre cumplimiento de la Ley, responsabiliza a los prestadores del cumplimiento de los derechos establecidos en ella, entre los cuales se encuentra el derecho de informar y el derecho al consentimiento informado. Agrega además que del incumplimineto se puede reclamar, además, en la Superintendencia de Salud. Cabe destacar que en esta situación se contempla un procedimiento de mediación y si no se arriba a una solución, es posible accionar judicialmente a través de las acciones civiles ordinarias.

Parte de la regulación sobre la temática se encuentra en el Reglamento sobre entrega de información y expresión de consentimiento informado en las atenciones de salud, contenido en el Decreto 31 del Ministerio de Salud y adoptado en 2012. Este texto jurídico en algunas casos reitera las disposiciones contenidas en la Ley 20.584, en otras las complementa, mientras que en otros pocos casos establece una regulación nueva.

Así, su art. 4 establece el derecho a ser informado, respecto de cualquier evento adverso que lo afecte, cualquiera sea la magnitud del daño que le hubiera ocasionado.

$\mathrm{Su}$ art. 6 obliga a que el lenguaje utilizado tenga en consideración el estado de salud y características socioculturales del paciente. 
El artículo 12 reproduce en gran medida el artículo de la Ley, pero agrega que la reglamentación especial que regule la materia debe incluir las modalidades que sean necesarias de adoptar en casos que el médico tratante (debe entenderse como profesional de la salud) determine que no está en condiciones de recibirla directamente o que padece de dificultades de entendimiento o alteraciones de conciencia que le impiden comprenderla.

$\mathrm{El}$ art. 14 indica que los prestadores individuales de salud y todo el personal en caso de prestadores institucionales, debe portar una credencial de identificación, visible y de fácil comprensión por parte de los usuarios, en la cual se establezca su nombre y apellidos, función e institución a la que pertenecen.

El art. 16 establece ciertas materias nuevas. Parte indicando que la forma y mecanismos para obtener el consentimiento informado de una persona en estado terminal estarán establecidos en un reglamento especial sobre la materia. Indica asimismo que si el tratante estima que la decisión del paciente o representante le expone a graves daños de su salud o riesgo de morir, riesgos evitables con tratamiento, debe recurrir al comité de ética correspondiente, mismo procedimiento a utilizar en casos de que la insistencia de indicación médica o de limitación de esfuerzo terapéutico sea rechazada por el paciente o su representante.

$\mathrm{El}$ art. 17 establece el derecho a pedir el alta voluntaria cuando se desee rechazar o interrumpir el tratamiento o medicamentos prescritos.

El artículo 19 señala que las personas que presenten una enfermedad mental debidamente diagnosticada o en proceso de evaluación diagnósti$\mathrm{ca}$, tienen los mismos derechos y deberes que las personas estándar, agregando que en el caso de presentar, además, una condición de discapacidad psíquica o intelectual, se regirán adicionalmente y en lo pertinente por la reglamentación especial sobre salud mental.

El art. 21 indica que las personas tienen el derecho de efectuar consultas y reclamos, debiendo regularse esta posibilidad en los reglamentos internos de cada establecimiento.
Por otra parte, en el ámbito de la investigación clínica en humanos, regulada principalmente en la Ley 20.120 sobre la investigación científica en el ser humano, su genoma, y prohíbe la clonación humana, de 2006, y su Reglamento, contenido en el Decreto 114 del Ministerio de Salud del año 2010, es necesario que se cuente con un consentimiento informado firmado, que incluya un anexo de revocación, para participar de una investigación. Este consentimiento debe, además, ser validado por un comité ético científico acreditado por la autoridad sanitaria.

\section{La praxis odontológica}

Los odontólogos "son para la sociedad expertos en conocimientos elevados y habilidades específicas, comprometidos individual y colectivamente a dar prioridad al bienestar de sus pacientes. Consecuentemente, cuando alguien se recibe de odontólogo toma un compromiso con la comunidad y acepta las normas y obligaciones de su profesión" (17). Entre estas obligaciones se encuentra dar cumplimiento al derecho a la información y a un consentimiento informado en los términos indicados anteriormente.

Ahora bien, el odontólogo ejerce una disciplina de servicio y auxilio, incorporando necesariamente la responsabilidad social e individual y que se realiza con independencia de la efectividad técnica lograda(18), pues se trata, en último término, de una obligación de medios y no de resultados.

En el ejercicio de la Odontología, especialmente en la mayoría de los procedimientos terapéuticos habituales, son utilizados biomateriales que optimizan algunos resultados clínicos. Estos pueden tener diversos tipos de origen en cuanto a su obtención, tales como: injertos óseos que son obtenidos del propio paciente (autólogos o autoinjertos); injertos óseos que proceden de individuos de la misma especie pero genéticamente diferentes (aloinjertos); injertos óseos de otra especie (xenoinjertos) y los injertos óseos fabricados sintéticamente (aloplásticos). En esta clase de procedimientos es común que los odontólogos tomen decisiones clínicas basadas en su experiencia clínica y en sus ámbitos de especialización, y es posible que, a menudo, omitan informar a sus pacientes sobre el origen de estos biomateriales y las venta- 
jas o desventajas que puedan tener en las diferentes aplicaciones terapéuticas. Así, por ejemplo, si el odontólogo usa el concepto de "injerto óseo", éste puede englobar perfectamente a todos los tipos de injertos; sin embargo tal concepto no especifica las características de su origen de obtención. Lo anterior puede parecer de poca importancia, no obstante presenta una enorme significación en caso de personas que profesan una religión o pertenecen a culturas determinadas que les impiden recibir injertos óseos de otros seres vivos.

En un sentido similar, se ha informado que los aloinjertos y xenoinjertos producen tasas de rechazo más altas entre las preferencias de los pacientes; mientras que, por el contrario, los injertos aloplásticos y autólogos son los injertos óseos más aceptados(19). Pese a lo anterior, pueden existir diferencias considerables en la efectividad de una terapia de acuerdo con un determinado tipo de injerto usado; por lo tanto, es importante una buena comunicación entre el especialista y el paciente de acuerdo con el rendimiento clínico esperado, de modo que el paciente posea toda la información que le permita ponderar la efectividad de un determinado proceso terapéutico, frente a una eventual renuncia al ejercicio del derecho a la libertad de conciencia o religión.

Existen estudios que sostienen cierto nivel de resistencia por parte de los odontólogos, particularmente en relación a la escrituración del consentimiento informado. Esto puede deberse a diversos factores, tales como desconocimiento del marco legal(16); poca relevancia o falta de implicación(5); falta de formación ética por parte de las universidades(9), pero también es posible atribuirlo a resabios de un enfoque paternalista, en el cual el paciente es solo receptor pasivo de los conocimientos del profesional.

Los odontólogos se han sensibilizado con la utilización del consentimiento informado, principalmente por el aumento de casos en que han sido demandados y por las sentencias negativas en su contra, debido a la ausencia de consentimiento informado(17). De este modo, los beneficios de la utilización del consentimiento y su escrituración suelen plantearse solo desde una perspectiva judicial. Así, se ha sostenido que tal utilización evita malos entendidos; que los pacientes con que se utiliza serían menos propensos a demandar y que provee la mejor vía de defensa contra demandas de los pacientes(10).

La relación entre demandas judiciales y derecho a la información puede verse reflejada en que, según estudios chilenos, la principal causa de reclamos en el procedimiento de mediación ante la Superintencia de Salud fue la de problemas de comunicación, seguida por la generación de falsas expectativas, ética profesional deficiente, entre $\operatorname{otras}(20)$.

Ahora bien, a este aumento de demandas judiciales se ha atribuido el encarecimiento de la atención y, especialmente, la aparición de la "medicina defensiva", entendida como decisiones médicas adoptadas más por temor a los reclamos o solicitudes de los pacientes, que por motivos clínicos(20).

Las circunstancias descritas dejan en evidencia que no es posible entender el consentimiento informado solo como una obligación jurídiconormativa, sino que debe ser entendido, también desde una perspectiva ética(5) y filosófica, en cuanto contribuye a comprender de manera completa lo que significa, para la libertad de las personas, el procedimiento por el cual estas se informan y toman sus propias decisiones(9). Este enfoque implica afirmar, sin lugar a dudas, que los contenidos establecidos en la Ley 20.584 y en el Decreto Supremo 31 no son sino mínimos u orientaciones, puesto que el proceso de consentimiento informado debe comprender todo lo necesario para permitir al ser humano ejercer su libertad.

Solo de esta comprensión amplia es posible recuperar el humanismo en la atención de salud oral, así como promover el respeto a la dignidad que merece todo paciente como fundamento del proceso comunicativo y deliberativo(7).

\section{Conclusiones}

Con lo dicho es posible sostener que el deber de informar - y específicamente en consentimiento informado- es importante desde múltiples perspectivas, no solamente desde la necesidad de dar cumplimiento a la normativa vigente. 
En la actualidad, aunque todos los modelos de consentimiento informado, en mayor o menor medida, parten de un enfoque bioético, las variables sociodemográficas, culturales y religiosas de los pacientes deben ser consideradas, pues se trata precisamente de obligaciones no escritas provenientes de la ética.

Pareciera que una forma conveniente de incorporar el enfoque ético en el proceso de consentimiento informado consiste en que forme parte de la formación curricular en las universidades, puesto que su utilización se realiza desde la formación de un estudiante de pre o posgrado en determinadas acciones terapéuticas que serán ejecutadas bajo supervisión de tutores clínicos, hasta las investigaciones ejecutadas por profesores.

Por otra parte, aunque el artículo 14 de la Ley 20.584 señala que el proceso de consentimiento se efectúa, generalmente, de manera verbal, muchos odontólogos tienen como práctica escriturar esta información, debido que esto genera mayor seguridad al profesional. Sin embargo, en ocasiones, no se considera en el consentimiento informado la realización de acciones que involucran los procedimientos previos a la terapia, tales como la indicación de radiografías o realización de guías operacionales o quirúrgicas, o la relación de exámenes complementarios que permitirán una planificación adecuada del tratamiento. En este sentido, es necesario informar al paciente no solo de la propuesta terapéutica enfocada en la solución de su problemática clínica, sino también del proceso de anamnesis odontológica.
Es posible sostener que falta una regulación más específica en relación con el consentimiento informado en Odontología, puesto que no está establecido en la legislación que se informe, por ejemplo, sobre el tipo del biomaterial que será usado en un determinado procedimiento terapéutico; sus características en cuanto a su origen u obtención; las implicancias de los posibles fracasos de la integración de este biomaterial en el sitio donante y las limitaciones del rendimiento clínico de estos productos.

Tal falta solo puede suplirse con un consentimiento informado más detallado, entendido en sus dimensiones éticas y filosóficas, es decir, que no pierda de vista que su fundamento es la libertad del ser humano. Solamente mediante esta concepción del consentimiento informado será posible asegurar que no se vulneren los derechos fundamentales de los pacientes. 
La obligación de informar y el consentimiento informado en Odontología - Estefanía Esparza-Reyes et al.

\section{Referencias}

1. Otero-Ruíz E. Acuerdos y normas nacionales e internacionales sobre ética médica y bioética. Revista Persona y Bioética 2000; 11-12: 118-122.

2. Chelouche T, Brahmer G. Bioética y Holocausto. Haifa: UNESCO; 2012.

3. Simón Lorda P. El consentimiento informado: Historia, teoría y práctica. Madrid: Editorial Triacastela; 2000.

4. Simón Lorda P, Concheiro Carro L. Consentimiento informado: teoría y práctica (I). Med Clin (Barc) 1993; 100(17): 659-663.

5. Hernández Gutiérrez P. M. Consentimiento informado en Odontología. Tesis para optar al grado de Doctor. Universitat de Valencia, 2015 (inédita).

6. Palomer L. Consentimiento informado en odontología. Un Análisis teórico-práctico” Acta Bioethica, 2009; 15(1): 100105.

7. García A. Consentimiento informado: aspectos éticos y legislación en la odontología. Humanidades Médicas 2013; 13(2): 393-411.

8. Emanuel EJ, Emanuel LL. Cuatro modelos de la relación médico-paciente. En Couceiro A. (coord.). Bioética para clínicos. Madrid: Editorial Triacastela; 1999: 109-126.

9. Miguel R, Zemel M. El consentimiento informado en odontólogos residentes de la Facultad de Odontología de la Universidad Nacional de La Plata. Acta Bioethica 2006; 12(1): 81-89.

10. Azzeddin R. El odontólogo frente al consentimiento informado. Aspectos relevantes. Acta Odontológica Venezolana 2011; 49(2): 1-7.

11. Ortíz A, Burdiles P. Consentimiento informado. Rev. Med. Clin. Las Condes 2010; 21(4): 644-652.

12. Sanz A, Del Valle, ML, Fernández M, Ferreira R. Teoría y Práctica del consentimiento informado. Cuadernos de Bioética, 2016; XXVII(1): 69-78.

13. De la Maza I. Consentimiento informado, una visión panorámica. Revista Ius et Praxis 2010; 16(2): 89-120.

14. Wahl J. Ficha clínica, uso de protocolos de seguridad y calidad, y consentimiento informado. Problemas de responsabilidad civil. En VVAA. Derechos y deberes de los pacientes. Estudios y textos legales y reglamentarios. Santiago de Chile: Universidad del Desarrollo; 2014: 16- 21.

15. Yágüez R. Prólogo. En Galán JC. Responsabilidad médica y consentimiento informado. Madrid: Editorial Civitas; 2001: I

16. Escobar MT. Consentimiento informado en Odontología. Estudio histórico-interpretativo en Cundinamarca 1990 2010. Revista Colombiana de Bioética 2014; 9(1): 7-125.

17. Torres-Quintana MA, Romo F. Bioética y ejercicio profesional de la Odontología. Acta Bioethica 2006; 12(1): 65-74.

18. Azzeddin R, Spina M, Tosta E. Dimensión ética en la práctica odontológica. Visión personalista. Acta Bioethica 2010; 16(2): 207-210.

19. Fuentes R, Bucchi C, Navarro P, Beltrán V, Borie E. Bone grafts utilized in dentistry: an analysis of patients' preferences. BMC Med Ethics 2015; 16(1): 1-6.

20. Portela FJ. Caracterización de los aspectos bioéticos de los reclamos realizados ante la Superintendencia de Salud de Chile en el área de Odontología. Acta Bioethica 2013; 18(1): 143-152.

Recibido: 10 de mayo de 2020

Aceptado: 3 de junio de 2020 